\title{
Transient Stability Enhancement of a Power System Considering Integration of FACT Controllers Through Network Structural Characteristics Theory
}

Akintunde Alayande ${ }^{1}$, Somefun A.O ${ }^{1}$, Tobiloba Somefun ${ }^{*}, 2$, Ademola Ademola ${ }^{2}$, Claudius Awosope ${ }^{3}$, Obinna Okoyeigbo ${ }^{2}$, Olawale Popoola $^{4}$

${ }^{1}$ Department of Electrical and Electronics Engineering, University of Lagos, Akoka, Yaba, 220282, Nigeria

${ }^{2}$ Department of Electrical and Information Engineering, Covenant University, Ota, 112107, Nigeria

${ }^{3}$ Department of Electrical Engineering, Kanni Samab Consultants, Ilupeju, 100252, Nigeria

${ }^{4}$ Department of Electrical and Electronics Engineering, Tshwane University of Technology, Pretoria, 0008, South Africa

A R T I C L E I N F O

Article history:

Received: 18 September, 2020

Accepted: 21 December, 2020

Online: 16 February, 2021

Keywords:

Network structural

characteristics theory

FACTS devices

Transient stability

Eigenvalue

Swing equation

\begin{abstract}
A B S T R A C T
Modern power systems are topologically and structurally complicated due to their complex interconnections. Consequently, the complexity of the dynamic stability assessment be-comes more tedious, most especially, when considering a power electronics-based power system operating under faulty conditions. This paper, therefore suggests an alternative approach of Network Structural-Based Technique (NSBT) for the analysis and enhancement of transient stability of a power system considering Flexible Alternating Current Transmission Systems (FACTS) devices integration. The mathematical formulations based on the NSBT as well as the dynamic swing equations, required for carrying out the stability analysis, are presented. The structural characteristics of the network are captured by considering the interconnections of the network elements and the impedances between them. The eigenvalue analysis is then explored to identify suitable and possibly weak load node locations where the influence of FACTS device placement within the network, could be most beneficial. The transient stability analysis before and after critical outage conditions is investigated. The transient stability of the network operating under critical outage condition is then enhanced considering the integration of a multi- UPFC controller, which is suitably located as identified by NSBT. The effectiveness of the suggested approach is tested using the modified standard IEEE 5-bus, 30-bus networks as well as the practical Nigerian 28-bus grid incorporating a multi-FACTs controller. The results obtained show that the FACTS device contributes significantly to improving the transient stability of a multi-FACTS-based power network. The information provided by this study is highly beneficial to the system operators, utilities investors and power engineers, most especially, for predicting system collapse during critical outage conditions.
\end{abstract}

\section{Introduction}

The dynamic stability assessment of most practical power systems, which are topologically weak, has been a major concern to most power system engineers and researchers in recent times [1]. This becomes inevitable as a result of the ever-increasing demand for electric energy, which is causing the system to operate close to its capacity limits. In order to avoid system collapse

*Corresponding Author: Tobiloba Somefun, Email:

tobi.shomefun@covenantuniversity.edu.ng

www.astesj.com

https://dx.doi.org/10.25046/aj0601107 without sacrificing the integrity of the network, it is expected that economic efficiency and the reliability of the network are maintained [2]. With the aim of achieving these, the power networks are faced with challenges such as overloading, voltage instability, and excessive transfer of power along transmission lines, to mention a few $[3,4]$. Moreover, the frequent voltage collapse recorded in most modern power system networks is due to the fact that the networks are structurally weak [5]. This effect could be as a result of the reactive power deficit in the network. It 
could also be traced to networks with very high resistance to reactance ratio, which is a characteristic of a distribution network or a radial transmission network. According to authors in reference [5], the least eigenvalue is approximately zero, and the magnitude of the elements of eigenvector associated to the least eigenvalue are approximately equal [5]. This constant magnitude shows that the network is a topologically weak power system, and it is due mainly to a wide relative electrical distance gap between the network load buses. A topologically weak network cannot be loaded up to its full capability limit without yielding a lower power transfer capability, and most power systems exhibit these structural characteristics, which make them be operated close to or beyond their permissible operating limits [6]. Therefore, there is a need for adequate compensation in order to enhance the transient stability of topologically weak power systems operating under critical contingencies and thereby enhancing the transfer capability of the network. This paper, therefore, attempts to provide an alternative approach to the enhancement of the transient stability of structurally weak power networks, which are operating under fault conditions considering the integration of FACTS devices.

Several contributions have been proposed by different authors for improving the transient stability of power systems undergoing disturbances through the integration of these FACTS devices [3]. FACTS devices are unique for their ability to preserve synchronism of generators whenever a major fault occurs within the network [7]. However, in order to obtain optimum performance of these FACTS devices, it is important to ensure that they are optimally placed within the power network under consideration. Traditionally, the problem is usually formulated as an optimisation problem, which is iterative in nature. For instance, the Modified Salp Swarm Optimization Algorithm (MSSOA) is used in [8] for the location of the UPFC (Unified Power Flow Controller. Another method proposed is the Particle Swarm Optimization algorithm in [7] for the optimal location of the STATCOM. Authors in reference [1] proposed a scheme using the zero dynamic approach to control the Thyristor Controlled Series Capacitors (TCSC) for its optimal location. The scheme controls the TCSC to investigate the transient stability of a multi-machine power system. The authors of [9] also present a hybrid BBO-DE algorithm to examine the performance of the system using the Static Var Compensator (SVC) and Power System Stabilizer (PSS). However, these iterative-based solution methods are not without challenges. The problem is formulated as a non-linear problem, which is a bit difficult to solve. Other problems include the existence of local minimal, non-convergence, time and space complexities etc. This influences to a greater extent, the results obtained, most especially, when dealing with a large practical network with multiple of contingency situation.

In this paper, a non-iterative method which solely depends on the structural interconnections of the network elements and their impedance values is suggested. This is because the study has shown that obtaining an effective solution to the problem actually lie in the structural interconnections of the network elements [10]. The main merit of this approach lies in the fact that the problem formulation as a linear and simple based on the fundamental circuit theory laws. Consequently, the solution is obtained in just one computation time. Hence, time and space complexities issues are totally eliminated. Also, the problem associated with the slack bus identification in the iterative-based methods is totally avoided.
Among the available FACTS controllers, UPFC has some inherent characteristics that are highly beneficial to solving some power system operational problems. For example, it can be used to control the flow of both active and reactive powers independently. Another benefit that could be derived from using UPFC is that it is a good controller for regulating the network load node voltages. These benefits are explored in this paper.

It has been shown, in the existing study that obtaining an effective solution to most power system problems does not depend on the loading conditions of the system but actually lie in the structural interconnections of the network elements [10]. Consequently, in this paper, a non-iterative method which solely depends on the structural interconnections of the network elements and their impedance values is proposed. The contributions of this paper to the active stream of research are as follows: The existing methods formulates the problem considered in this paper as an optimization problem which are without drawbacks such as local minimal issues, divergence problem etc. This present study, however, reformulates the problem of identifying UPFC placement as a linear problem based on the fundamental circuit theory laws. Consequently, the solution is obtained in just one computation time. Hence, time and space complexities issues are totally eliminated. Also, the problem associated with the slack bus identification in the iterative-based methods is totally avoided.

The remaining parts of the paper are structure as follows: section 2 presents the theoretical framework as well as the mathematical formulations to the problem, in section 3 , the descriptions of the networks used are presented. The programming tools used are also presented in section 3. Section 4 presents the numerical results obtained and the discussion of the results. The paper is concluded in section 5 .

\section{Theoretical Background and Mathematical Formulations}

This section provides the mathematical formulations and methodology used in building the numerical approach for the study. In this paper, the Newton-Raphson method used in carrying out the load-flow analysis is briefly revisited, the derivation of swing equation is presented, the mathematical formulation of the non-iterative dependent approach for identifying suitable load nodes where FACTS Devices could be placed for enhancement of the transient stability is presented, and the formulation for enhancing a structurally weak network is presented.

\subsection{Static and Dynamic Modelling for Transient Stability Assessment}

The steady-state analysis of power system is usually studied by solving the static power flow equations. Various approaches to solving these equations abound in the literature. However, the prominent solution techniques include Gauss-Seidel method [11], Fast Decoupled method and Newton - Raphson method [11, 12]. For the purpose of this study, the Newton - Raphson method is used because of its fastest rate of convergence as well as its selfcorrecting mechanism [13]. The complex power equations at any node $i$ of an n-bus power system can easily be expressed as [14].

$$
P_{i}+j Q_{i}=\dot{V}_{i} \sum_{j \in i} \hat{Y}_{i j} \widehat{V}_{J}
$$




$$
\begin{aligned}
& \forall j \in i(i=1,2, \ldots, n) \\
& \dot{V}_{i}=V_{i} e^{j \theta_{i}}
\end{aligned}
$$

where $V_{i}$ and $\theta_{i}$ are the magnitude and phase angle of voltage at bus $i$.

The elements of admittance matrix can be expressed as

$$
Y_{i j}=G_{i j}+j B_{i j}
$$

From (1), the real and reactive power at any bus $i$ respectively can be formulated as

$$
\begin{aligned}
P_{i}= & V_{i} \sum_{j \in i} \widehat{V}_{J}\left(G_{i j} \cos \cos \theta_{i j}+j B_{i j}\right. \\
& \left.\sin \sin \theta_{i j}\right) \\
Q_{i}= & V_{i} \sum_{j \in i} \widehat{V}_{J}\left(G_{i j} \sin \sin \theta_{i j}-j B_{i j}\right. \\
& \left.\cos \cos \theta_{i j}\right)
\end{aligned}
$$

such that $(i=1,2, \ldots, n)$

where $\theta_{i j}=\theta_{i}-\theta_{j}$, which is the voltage phase angle difference between bus $i$ and $j$.

The result is a linear system of equations that can be expressed as follow:

$$
[\Delta \theta \Delta|V|]=J 1[\Delta P \Delta Q]
$$

where $\Delta P$ and $\Delta Q$ are mismatch equations given by (7) and (8) respectively as

$$
\begin{gathered}
\Delta P_{i}=-P_{i}+V_{i} \sum_{j \in i} \widehat{V}_{j}\left(G_{i j} \cos \cos \theta_{i j}+j B_{i j}\right. \\
\left.\quad \sin \sin \theta_{i j}\right) \\
\Delta Q_{i}=-Q_{i}+V_{i}\left(G_{i j} \sin \sin \theta_{i j}-j B_{i j}\right. \\
\left.\cos \cos \theta_{i j}\right)
\end{gathered}
$$

and $J$ is a matrix of partial derivatives known as a Jacobian given as

$$
\left[\frac{\partial \Delta P}{\partial \theta} \frac{\partial \Delta P}{\partial|V|} \frac{\partial \Delta Q}{\partial \theta} \frac{\partial \Delta Q}{\partial|V|}\right]
$$

The linearised system of equations is solved to determine the next guess $(m+1)$ of voltage magnitude and angles as follows:

$$
\begin{gathered}
\theta^{m+1}=\theta^{m}+\Delta \theta \\
|V|^{m+1}=|V|^{m}+\Delta|V|
\end{gathered}
$$

where $\mathrm{m}$ is the iteration count. The process is iterated with a common stopping condition where the mismatch result is less than a given tolerance, $\varepsilon$.

For effective evaluation of power system transient stability assessment, the rotor angle dynamics provides a helpful insight into the behaviour of the system, most especially, when a sudden disturbance occurs. The sudden occurrence of a disturbance in a power system usually causes an increase in the mechanical torque $\left(T_{m}\right)$ of the prime mover, and an acceleration torque $\left(T_{a}\right)$ is developed if the mechanical torque is greater than the electromagnetic torque $\left(T_{e}\right)$. Mathematically,

$$
T_{a}=T_{m}-T_{e}
$$

The machine is accelerated with an inertia $J$ which consists of the inertia of the generator and prime mover. Therefore,

$$
\mathrm{J} \frac{d \omega_{m}}{d t}=T_{a}=T_{m}-T_{e}
$$

where $t$ is time in seconds and $\omega_{m}$ is the angular velocity of the rotor in mechanical rad/s. Expressing $J$ in terms of inertia constant $\mathrm{H}$ results to

$$
J=\frac{2 H}{\omega_{0 m}^{2}} V A
$$

Therefore,

$$
\frac{2 H}{\omega_{0 m}^{2}} V A \frac{d \omega_{m}}{d t}=T_{m}-T_{e}
$$

Introduction of a new angular velocity of the rotor, $\omega_{r}(\mathrm{rad} / \mathrm{s})$ results to

$$
2 H \frac{d \underline{\omega}_{r}}{d t}=\underline{T}_{m}-\underline{T}_{e}
$$

Alternatively,

$$
\frac{d \underline{\omega}_{r}}{d t}=\frac{d^{2} \delta}{\omega_{0} d t^{2}}
$$

where $\delta$ is the angular position of the rotor (elect. $\mathrm{rad} / \mathrm{s}$ ) with respect to a synchronously rotating reference frame.

By combining (16) and (17), the dynamics model of the rotor angle known as the swing equation can, therefore, be easily obtained expressed as

$$
\frac{2 H}{\omega_{0}} \frac{d^{2} \delta}{d t^{2}}=\underline{T}_{m}-\underline{T}_{e}
$$

\subsection{Identification of Suitable Load Nodes for Facts Device Placement: A Network Structural Perspective}

The mathematical formulation for the problem considered in this paper is viewed from the fundamental circuit theory laws perspective. This is employed to develop the inherent structural 
properties of power network considering the interconnectivity of the network elements and the impedance between them [15]. Though this theory has been extensively applied to solve various power system problems in the literature [16-19], there are more to the theory than we have had in the past. For instance, the application of this topological-based concept in resolving transient stability issues has not been holistically investigated. This is the main focus of this paper.

First, let us consider a simple power system network, to depict the structural interconnections of various power network elements, as shown in Figure 1.

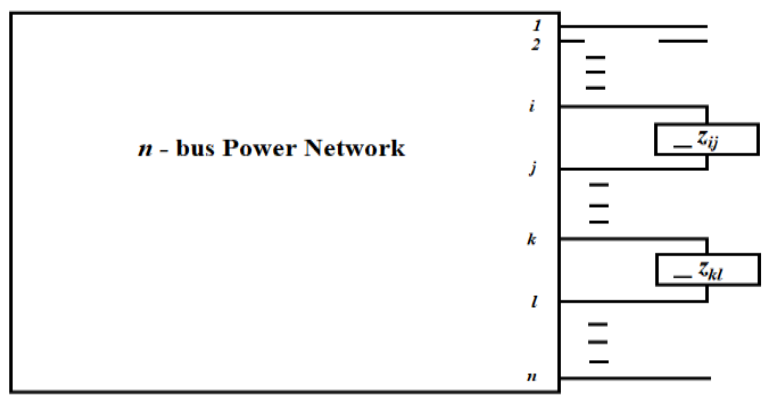

Figure 1: Interconnections of power network elements [4]

The matrix equation that relates the node voltages and line currents in terms of the line parameters can be written in its compact form as [5]

$$
\left[A_{c}\right]\left[V^{\prime}\right]=\left[\begin{array}{llll}
z_{i j} & 0 & 0 & z_{k l}
\end{array}\right]\left[\frac{I_{i j}}{I_{k l}}\right]
$$

where

$$
\left[V^{\prime}\right]=\left[V^{\prime}{ }_{1}, V^{\prime}{ }_{2}, \ldots, V^{\prime}{ }_{i}, V_{j}, \ldots, V^{\prime}{ }_{l}, \ldots, V^{\prime}{ }_{n}\right]
$$

where

$A_{c}$ is termed as the coupling matrix, $\left[V^{\prime}\right]$ represents the vector of the nodal voltage within the network after the enhancement through the identification of suitable nodes for placement of reactive power supports, $I_{i j}$ and $I_{k l}$ are the currents through the network branches $i-j$ and $k-l$ respectively.

For instance, the coupling matrix, based on Figure 1 can be formulated as

$$
\begin{aligned}
& {\left[A_{c}\right]=\left[\begin{array}{llllllll}
0 & 0 & 0 & \ldots & 0 & 0 & 1 & 0
\end{array}\right.} \\
& \begin{array}{llllllllll} 
& 1 . & 0 & 0 & 0 & 0 &
\end{array} \\
& -1 . . .000]
\end{aligned}
$$

Since a change in current causes a change in bus voltages, we can therefore write

$$
\left[V^{\prime}\right]=\left[Z_{l}\right]\left[l_{o}+\Delta l\right]
$$

Alternatively,

$$
\left[V^{\prime}\right]=[V]+[\Delta V]
$$

where $[V]$ represents the voltage profile of the original power network.

$[\Delta V]$ denotes the change in the vector of the voltage profile for the network before the enhancement, which can easily be expressed as

$$
[\Delta V]=-\left[Z_{l}\right]\left[A_{c}^{T}\right]\left[I_{i j} I_{k l}\right]
$$

From equation (24),

$$
\begin{aligned}
& {\left[\begin{array}{ll}
I_{i j} & I_{k l}
\end{array}\right]=\left[Y_{\text {bus }}\right]\left[V_{i}-V_{j} V_{k}-V_{l}\right]} \\
& {\left[Y_{\text {bus }}\right]=\left\{z^{\text {new }}+\left[A_{c}\right]\left[Z_{l}\right]\left[A_{c}^{T}\right]^{-1}\right\}} \\
& z^{\text {new }}=\left[\begin{array}{llll}
z_{i j} & 0 & 0 & z_{k l}
\end{array}\right]
\end{aligned}
$$

Obviously, the structural interconnections that exist between the network nodes of the enhanced network is fully captured in (26)

In order to investigate the influence of the network structural properties on the location of the FACTs devices for effective enhancement of the network, the eigenvalue decomposition approach is applied to (26) as [15]

$$
\left[Y_{\text {bus }}\right]=W \sum W^{T}=\sum_{i=1}^{n} \omega_{i} \rho_{i} \omega_{i}^{T}
$$

where $W$ and $W^{T}=n$-by $-n$ orthogonal eigenvectors of $\left[Y_{b u s}\right]$.

$$
\rho_{i}=\text { The eigenvalue at any bus } i, \omega_{i} \text { and } \omega_{i}^{T} \text { respectively. }
$$

The inverse relationship between the bus voltage and their respective eigenvalues can be expressed as

$$
[V]=\sum_{i=1}^{n} \frac{\omega_{i}^{T} \omega_{i}}{\rho_{i}}[I]
$$

Various methods have been identified to determine the eigenvalue of the power network. They are broadly classified under iterative and no-iterative based methods. More recently, network structural characteristics based approach presents a model which provides a clear distinction between generation and load buses, as presented in reference [19]. This is shown by formulating the generator voltage and the load current as functions of their respective generator currents and load voltages as

$$
\left[\begin{array}{ll}
V_{G} & I_{L}
\end{array}\right]=\left[Z_{G G} H_{G L} W_{L G} C_{L L}\right]\left[I_{G} V_{L}\right]
$$

where

$$
\begin{aligned}
Z_{G G} & =Y_{G G}{ }^{-1} \\
H_{G L} & =-Y_{G G}{ }^{-1} Y_{G L} \\
W_{L G} & =Y_{L G} Y_{G G}{ }^{-1} \\
C_{L L} & =Y_{L L}-Y_{L G} Y_{G G}{ }^{-1} Y_{G L}
\end{aligned}
$$

Obviously, from (30), it can be seen that the relationship between the vector of generator node voltages and the load node currents is governed by the matrix CLL, which captures the influence of all the interconnections of load buses after the influence of generator buses has been eliminated. Application of the eigenvalue presented in (29) can, therefore, be used to decompose CLL, which gives an inverse relationship between the load bus voltage and the associated eigenvalue. This relationship is used in this paper to identify the weak load nodes within the network [19]. 


\section{Numerical illustrations}

Case 1: The Standard IEEE 5-Bus Network

The standard IEEE 5- bus system network consists of twogeneration nodes and three load nodes, which are interconnected by seven transmission lines. The one-line diagram is presented in Figure 2 using the PSAT interface. The line data are adapted from reference [20] and are sampled for determining the eigenvalues of the nodes and their corresponding eigenvectors.

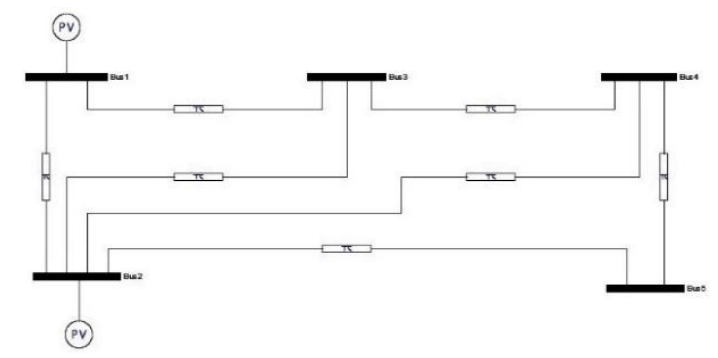

Figure 2: Single-line diagram of the standard IEEE 5-bus network

\section{Case 2: The Standard IEEE 30 - Bus Network}

Figure 3 shows the single line diagram, drawn in the PSAT environment, for the standard IEEE 30-bus network. This is used, as the second case study considered, in order to verify the effectiveness of the methodology suggested in this study. It comprises of six generator buses, twenty-four load buses, which are interconnected by forty-one transmission lines. In this study, the PSAT environment is used in conjunction with the MATLAB $2016 \mathrm{a}$ as the programming tools.

\section{Case 3: The Nigerian 28-Bus Network}

In order to investigate the effectiveness of the proposed approach in this study to practical power systems, the Nigerian 28bus is considered whose one-line diagram is shown in Figure 4. The network comprises ten generator buses, eighteen load buses, which are connected by thirty-one transmission lines. It worth noting that the sizing of the UPFCs used in this study is adapted from the work of Melodi [21].

\section{Results and Discussion}

This section presents and discusses the simulation results in this study obtained based on the approach suggested. Three power system networks are considered to verify the efficiency of the method. The standard IEEE 5-bus network is first considered, then the standard IEEE 30-bus system using PSAT simulation and then the Nigerian 28-bus system using MATLAB programming tools. The data used is on 100MVA base. The graphical representation as well as the tabular presentation of the results are displayed and explained. The transient stability of the networks before reinforcement and before the integration of UPFC are presented, which serve as the base case. These results are then compared with the transient stability, of the system, after the reinforcement and integration of UPFC.

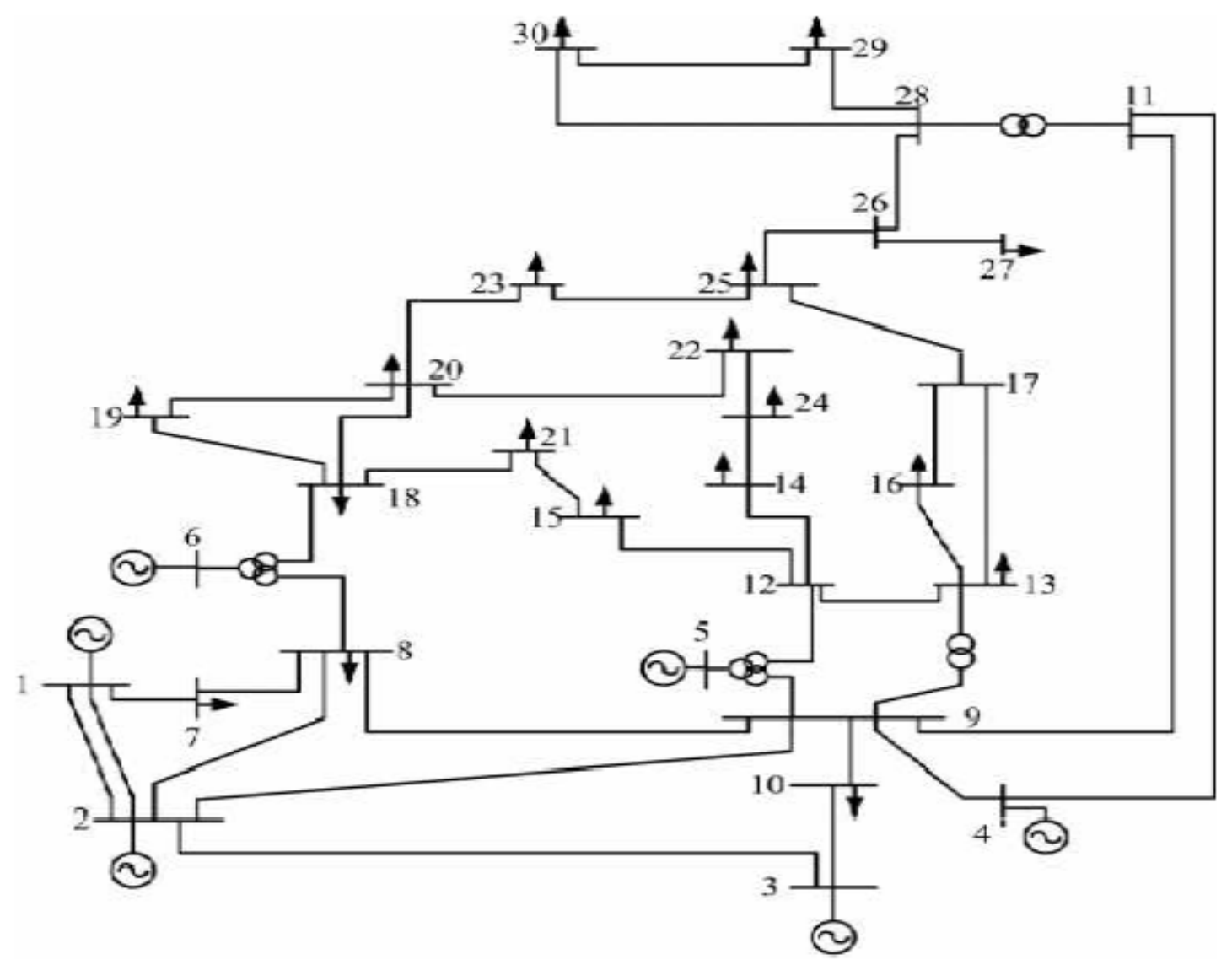

Figure 3: Single-line diagram of the standard IEEE 30-bus network 


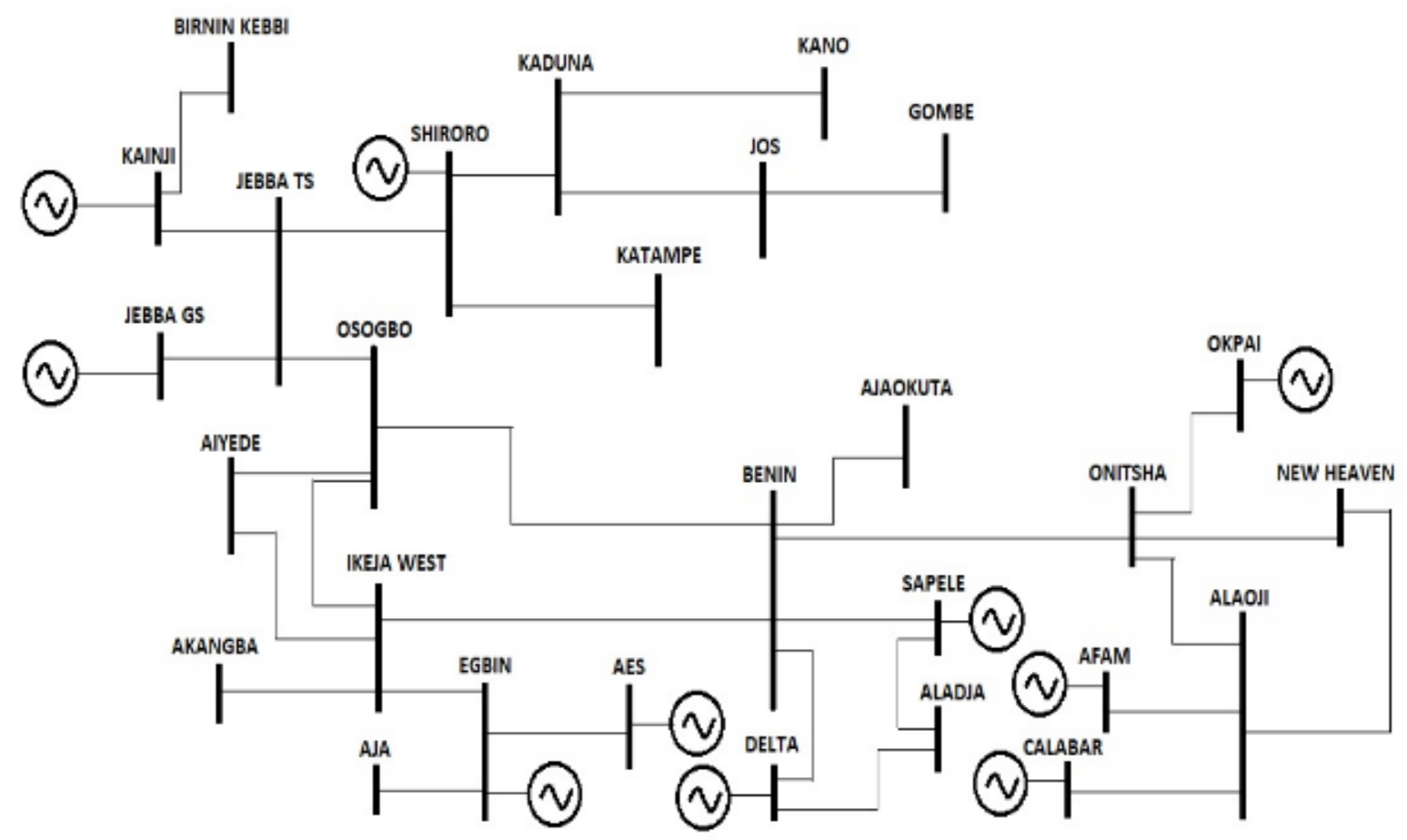

Figure 4: Single-line diagram of the Nigerian 28-bus network

\subsection{Investigation of The Network Strength and Identification of The Suitable Load Nodes for Network Reinforcement}

The eigenvalue analysis, on the reduced bus admittance of the network, based on the CLL matrix, is first performed. In this matrix, the interaction of the generators has been eliminated. So, the output of this matrix contains only the load-to-load interaction.

The IEEE 5-bus and the IEEE 30-bus networks are subjected to tests of determining the eigenvalues and the corresponding eigenvector, measured to confirm or ascertain the strength of the network. The network data are from reference [20]. The same procedures are extended to the Nigerian 28-bus network whose line data are adapted from reference [22]. The MATLAB 2016a is used as the programming tool to determine the eigenvalues and their corresponding eigenvectors in each case. It should be iterated that the sizing of the UPFCs employed in this study is carried out based on the existing work presented by the authors of reference [21]. The results obtained, for each case, are presented and discussed in the subsections that follow:

\section{Case 1: The Standard IEEE 5-Bus Network}

The modified network of the IEEE 5-Bus power network is shown in Figure 5. The transmission line where the multi-UPFC is located is first identified using eigenvalue analysis and then integrated into the network at the identified location, as shown in Figure 5. This UPFC is controlled such that appropriate compensation is delivered to the network, in steps, in order to maintain the network voltage profile such that the integrity of the network is maintained. This is carried out using the NewtonRapson power-flow-based technique. The load-flow analysis is performed in this study to determine the base case voltage profile of the two networks considered. Figure 6 shows the graphical representation of the voltage profile obtained for the power-flow analysis using the standard IEEE 5-bus system.

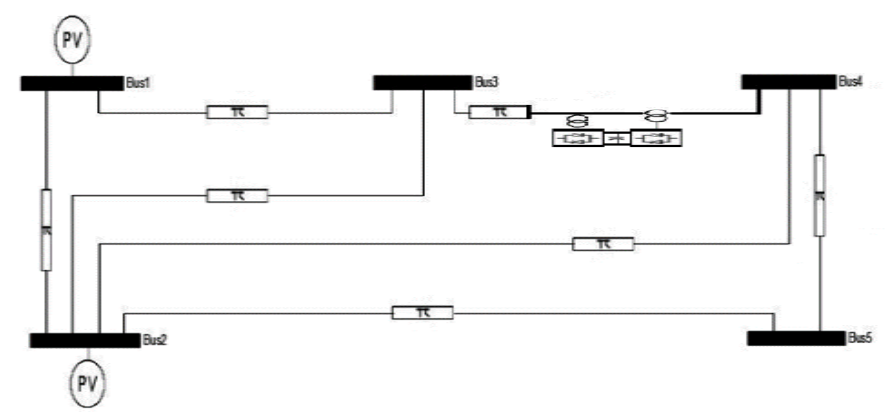

Figure 5: Single-line diagram of the modified IEEE 5-bus network

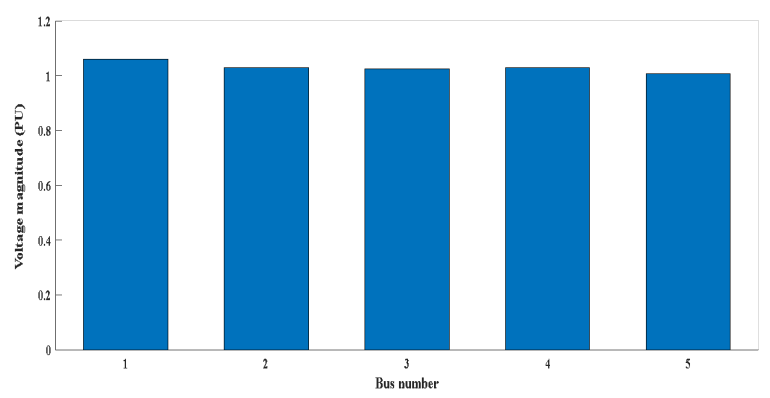

Figure 6: Base case voltage profile for the standard IEEE 5-bus network

The study then investigates the influence of FACTS device (UPFC) integration into the network during a disturbance. This is carried out by considering the transient stability of the network with and without FACTS. The simulation results for the two scenarios (with and without UPFC) are then compared. The results obtained from the eigenvalue analysis is ranked in ascending order, as shown in Table 1. The load node associated with the least eigenvalue is the suitable bus where the placement of the FACTS device would be of the highest benefit to the network. From Table 1, it can be seen that the least eigenvalue is ranked 1st, and this corresponds to load node 4 within the 
network. In other words, as evident from Table 1, Bus 4 has the least eigenvalue of 0.1120 , and this makes it the weakest bus in the system. This implies that the FACTS device will provide better compensation to the network if placed on the transmission line connecting buses 4 and 5. The eigenvector elements associated with the least eigenvalue of 0.1120 at bus 4 are found to be approximately equal to 0.571 , as shown in Figure 7 . This confirms the structural weakness of the network.

Table 1: Identification of a suitable location of FACTS device in the IEEE 5-Bus

\begin{tabular}{|c|c|c|}
\hline Load node & Eigenvalue & Ranking \\
\hline 4 & 0.1120 & $1^{\text {st }}$ \\
\hline 5 & 15.6029 & $2^{\text {nd }}$ \\
\hline 3 & 71.4702 & $3^{\text {rd }}$ \\
\hline
\end{tabular}

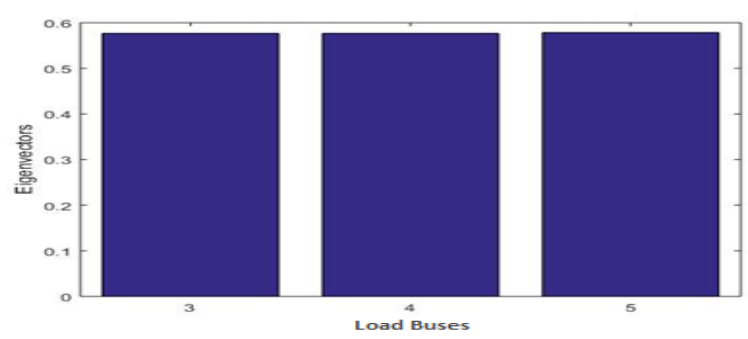

Figure 7: Eigenvectors associated with the least eigenvalue of the IEEE 5-bus network

\section{Case 2: Standard IEEE 30-Bus Network}

When the analysis of the eigenvalues is performed using the standard IEEE 30-bus power network, the transmission line, which is most suitable for placement of the multi-UPFC controller is determined. The modified network for the standard IEEE 30bus network, when the UPFC is integrated, using the PSAT interface is shown in Figure 8. The same procedures, as explained in the preceding section, are repeated for the standard IEEE 30bus network. The base case voltage profile using the standard IEEE 30-bus system is presented in Figure 9.

The same procedural steps followed in the case of the IEEE 5bus network are also followed using the standard IEEE 30-bus network for identifying the suitable load nodes where the reactive power supports should be located within the network. Similar simulations are also carried out using the standard IEEE-30 bus system. The ten buses with the least eigenvalues within the network are selected, ranked and presented in Table 2.

Bus 23 is the load bus associated with the least eigenvalue of 0.00216 , as shown in Table 2 . The corresponding eigenvectors for this least eigenvalue are found to be equal in magnitude with a value of 0.235 , as shown in Figure 10. This constant eigenvector magnitude shows that a very wide electrical distance exists between the load nodes, which accounts for the topological weakness within the network under consideration. This has a greater influence on the power-flow through the transmission lines within the network.

Table 2: Identification of a suitable location of FACTS device in the IEEE 30-Bus

\begin{tabular}{|c|c|c|}
\hline Load node & Eigenvalue & $\begin{array}{c}\text { Ranking of } \\
\text { eigenvalue }\end{array}$ \\
\hline 21 & 7.3285 & $10^{\text {th }}$ \\
\hline 22 & 6.7473 & $9^{\text {th }}$ \\
\hline 23 & 0.0216 & $1^{\text {st }}$ \\
\hline 24 & 0.7491 & $2^{\text {nd }}$ \\
\hline 25 & 5.5871 & $8^{\text {th }}$ \\
\hline 26 & 1.5335 & $3^{\text {rd }}$ \\
\hline 27 & 2.1872 & $4^{\text {th }}$ \\
\hline 28 & 4.5805 & $7^{\text {th }}$ \\
\hline 29 & 3.2186 & $6^{\text {th }}$ \\
\hline 30 & 3.0953 & $5^{\text {th }}$ \\
\hline
\end{tabular}

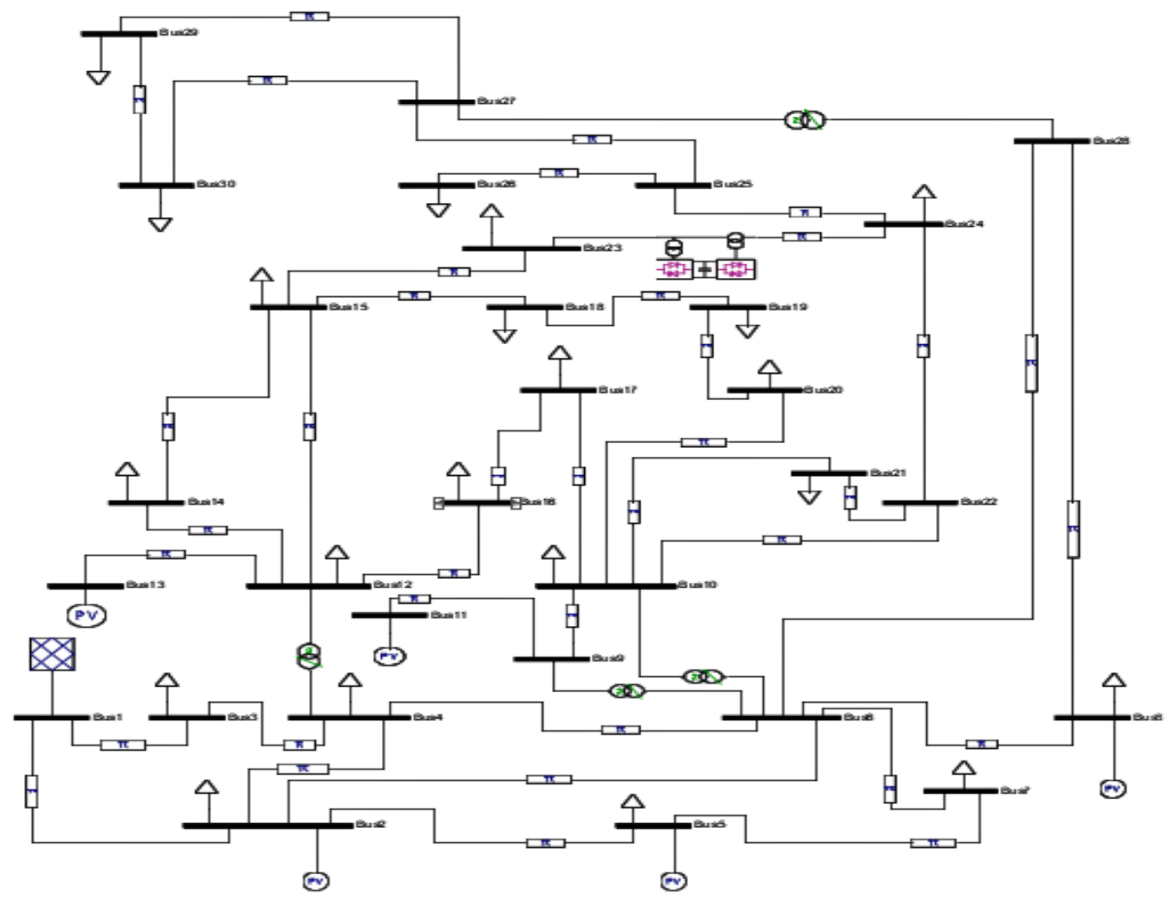

Figure 8: Single-line diagram of the modified IEEE 30-bus Network 


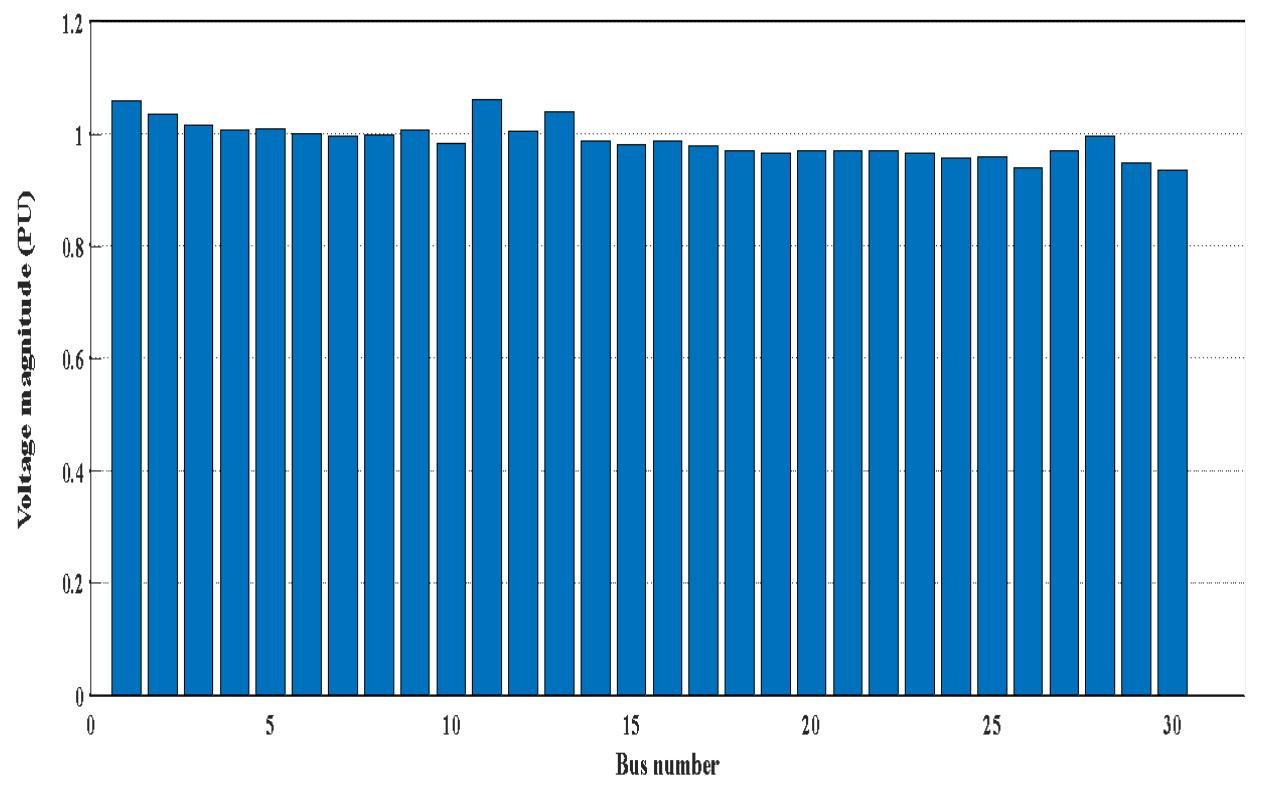

Figure 9: Base case voltage profile of the standard IEEE 30-bus network

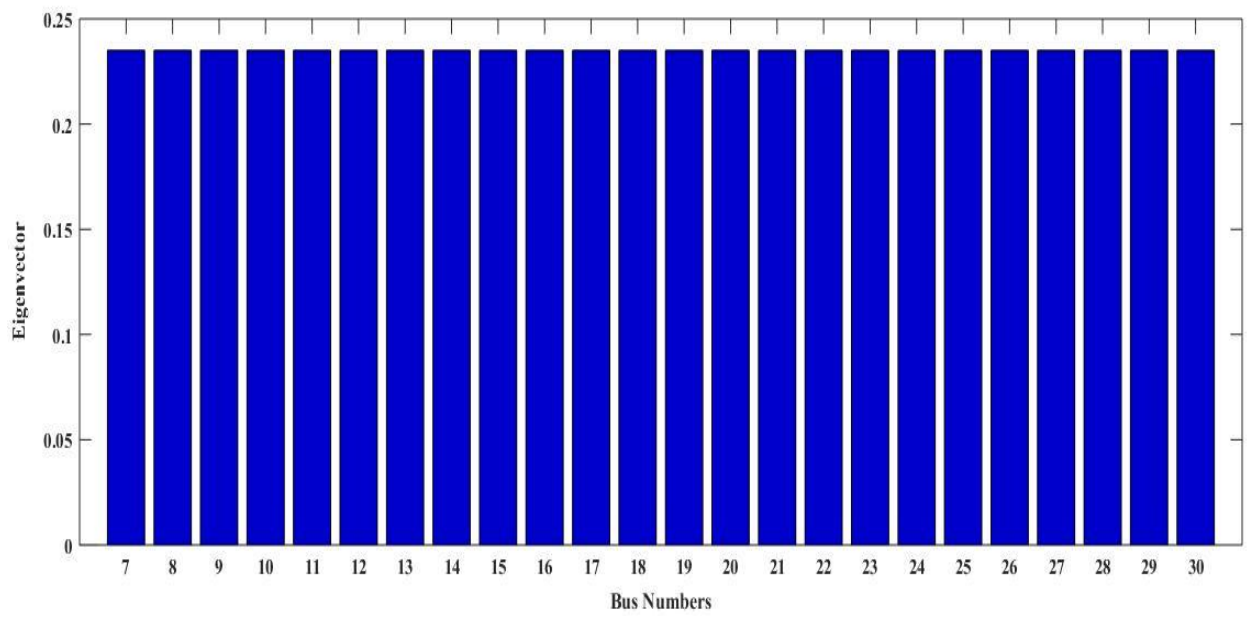

Figure 10: Eigenvectors of the least eigenvalue of the standard IEEE 30-bus network

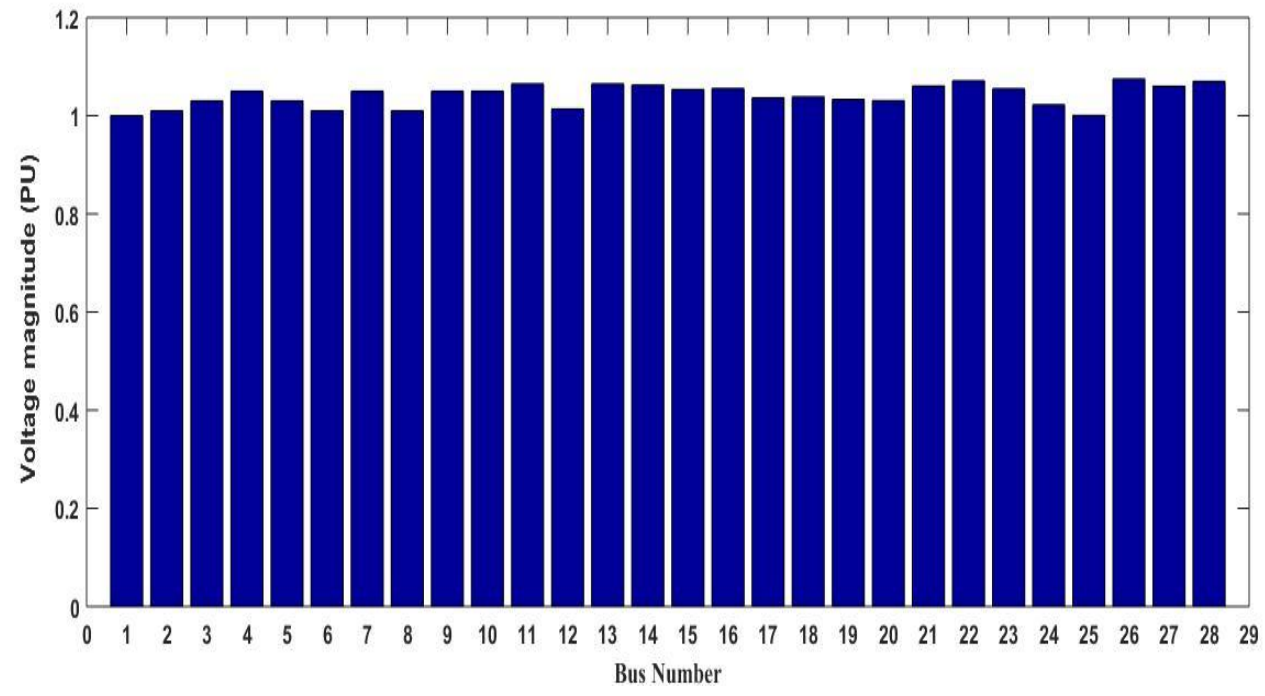

Figure 11: Base case voltage profile of the Nigeria 28-bus network 
Table 3: Identification of a suitable location of FACTS device in the Nigerian 28Bus

\begin{tabular}{|r|l|c|c|}
\hline $\begin{array}{l}\text { Load } \\
\text { node }\end{array}$ & Name & Eigenvalue & $\begin{array}{l}\text { Ranking of } \\
\text { eigenvalue }\end{array}$ \\
\hline 19 & Akangba & 143.3201 & $10^{\text {th }}$ \\
\hline 20 & Ikeja West & 103.4449 & $9^{\text {th }}$ \\
\hline 21 & Onitsha & 98.5211 & $8^{\text {th }}$ \\
\hline 22 & New Haven & 2.8253 & $1^{\text {st }}$ \\
\hline 23 & Alaoji & 3.8524 & $2^{\text {nd }}$ \\
\hline 24 & Aladja & 54.0964 & $7^{\text {th }}$ \\
\hline 25 & Aja & 19.7755 & $3^{\text {rd }}$ \\
\hline 26 & Birnin Kebbi & 32.3796 & $5^{\text {th }}$ \\
\hline 27 & Kaduna & 29.6417 & $4^{\text {th }}$ \\
\hline 28 & Kano & 51.5486 & $6^{\text {th }}$ \\
\hline
\end{tabular}

The results of the eigenvalues, which are associated with the network load buses, as presented in Table 3, are then ranked in increasing order from the least value to the largest value. It is revealed based on the results that the weakest node within the network is bus 22 (New Haven) whose eigenvalue is 2.8253 and the next to it is bus 23 (Alaoji), with the eigenvalue of 3.8524 . The implication of this is that a large electrical distance exists between the load buses 22 (New Haven) and 23 (Alaoji). This, therefore, suggests that the load bus 22 (New Heaven) is the weakest bus within the network. With this, the critical line is determined and the transmission line (New Haven - Alaoji), through which the UPFC is connected, is as therefore identified. The UPFC placement is, therefore, located between the buses 22 (New Haven) and 23 (Alaoji) as shown in the modified network shown in Figure 12. The eigenvectors that correspond to the least eigenvalue of 2.8253 is found to be approximately constant with a value of 0.2371 , and the results are shown graphically in Figure 13 in which all the load buses are seen to maintain equal values of eigenvector.

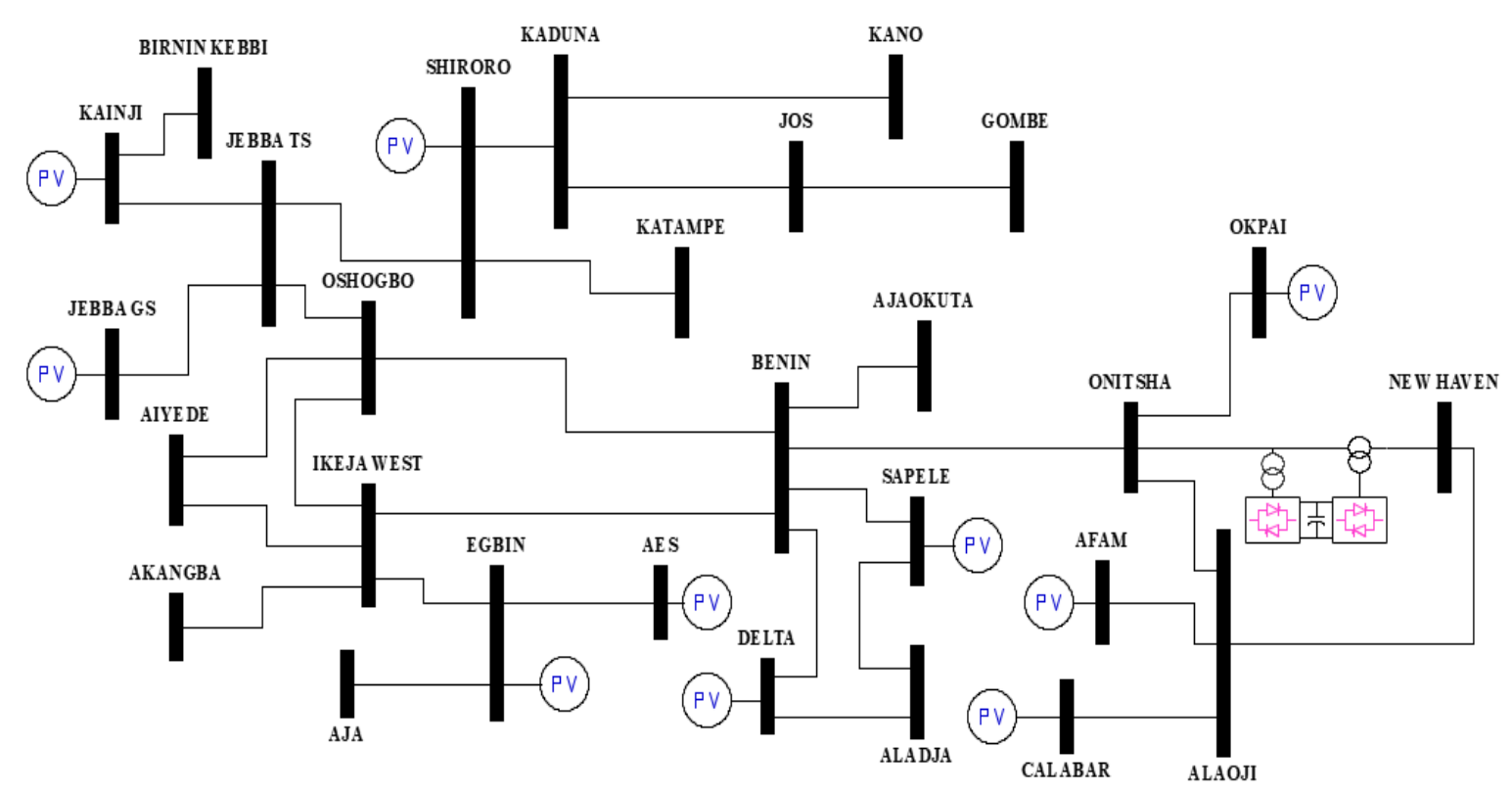

Figure 12: Single-line diagram of the modified Nigerian 28-bus Network

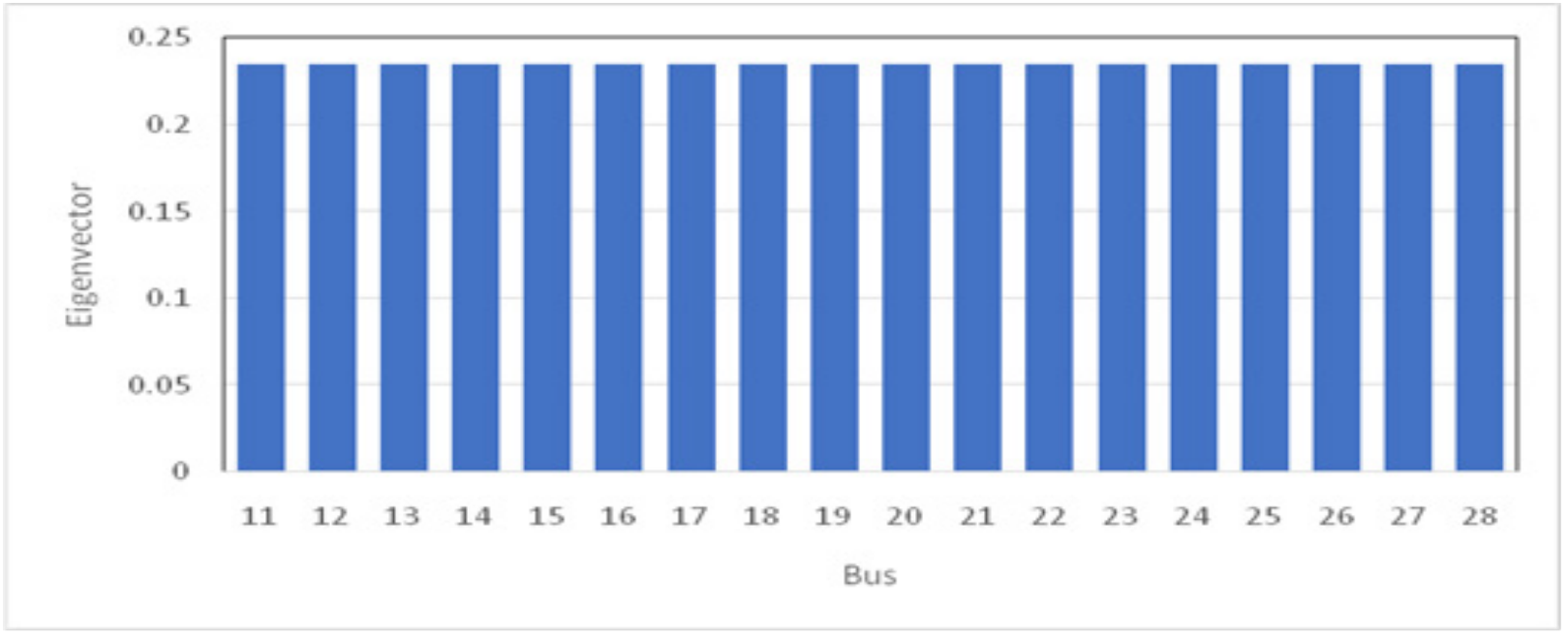

Figure 13: Eigenvectors corresponding to the least eigenvalue of New Heaven in the Nigerian 28-bus network 


\subsection{Transient Stability Assessment}

In this section, the assessment of the system transient stability, when subjected to disturbances, is investigated. The responsiveness of the power systems to the sudden occurrence of faults are assessed. This serves as the base case solution, and the results obtained are presented. Based on the phase difference and the angle of convergence, the time taken by the power systems to regain stability and return to a normal mode of operation is considered. The transient stability assessment, considering the reinforcement of the network through the integration of multiUPFC, is then carried out. The comparison of results is then made in order to investigate the impact of the FACTS device on the reinforcement of the topologically weak power networks. The results obtained from the simulations are presented in the sections that follow.

\section{Case 1: Transient Stability Assessment of The IEEE 5-Bus System}

This power network is subjected to a fault on bus 4 being the most critical load bus as identified based on the eigenvalue analysis. The contingency analysis is then performed by the removal of the transmission lines connected to it based on the N-1 criterion to determine the impact of the transmission line outages on the stability of the system. The results obtained from the simulations before the integration of the UPFC are shown in Figure 14.

Figure 14 (a) - (c) show the dynamic response for the transient stability assessment before the integration of UPFC for possible upgrading of the network. In Figure 14 (a), it can be seen that the effect of the disturbance during which line 2-4 is removed causes the rotor angle to fluctuate between the values of -2.50 and -22.50 . The phase angle after returning to synchronism, is found to become stable at -12.50 . However, the result of the transient assessment for the outages of line 3-4 and line 4-5 shows similarities in the phase angle difference, as shown in Figure 14 (b) and (c) respectively. The rotor angles fluctuate between -40 and -22.50 in both cases and the stable phase angle difference after returning to synchronism is -13.250 .

In order to investigate the impact of the multi-UPFC on the transient stability of the network, the transient stability assessment of the network is carried out considering the integration of the multi-UPFC into the network. From the eigenvalue results presented earlier in Table 1, suitable locations for the placement of multi-UPFC are identified based on the magnitude of the eigenvalues of the network load buses. It is seen that the bus with the least eigenvalue is identified as the most critical bus (bus 4) within the network. The transmission line connecting the most critical bus and the next ranked eigenvalue bus (bus 5) is identified as the most critical transmission line in the system. In other words, the most critical line in the IEEE 5-bus system, based on the approach suggested in this work, the critical line is the line connecting buses 4 and 5 . In this paper, we assumed the worst scenario and therefore suggested that when a fault occurs at the most critical bus, the line connecting buses 4 and 5 should be removed to clear the fault. Furthermore, as presented in Table 1, the next ranked eigenvalue bus after bus 5 is bus 3 . This implies that the network under consideration can easily be enhanced or upgraded by placing the multi-UPFC controller in series with the line which connects the most critical bus (bus 4 ) and bus 3 . It is noteworthy that the third line (line 2-5) is connected to bus 2 , which is a generator bus. This is not considered for the mutiUPFC as the generator is capable of reinforcing the line with the needed compensation.

After the integration of FACTS devices into the network, the same procedure carried out without the incorporation of UPFC is repeated. The reactive power is then improved in steps, and the transient stability of the system is analysed. The most significant variation is observed when the reactive load demand is compensated to $30.5 \mathrm{MVAR}$. The results obtained, considering the $\mathrm{N}-1$ criterion within the network is shown in Figure 15.

It is observed that an improvement in the phase angle difference occurs after integrating the UPFC between the lines. Also, the angle of convergence is seen to have been improved. Figure 15 shows that the phase angle difference is found between -4 and -20 and the angle of convergence after the system regains synchronism is -120 , which is a significant improvement from the base case examined.

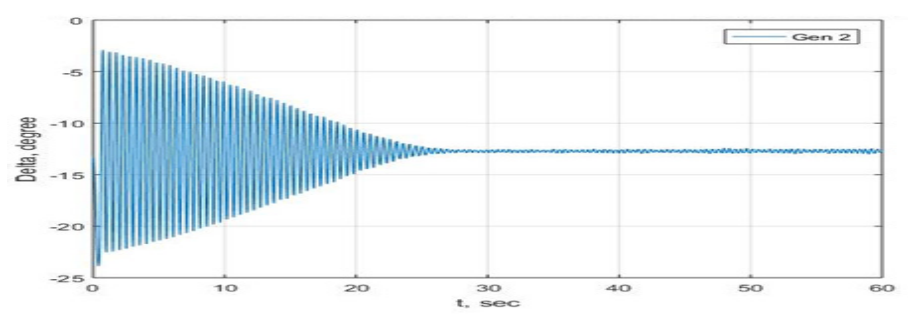

(a): Phase angle difference with line 2-4 removed

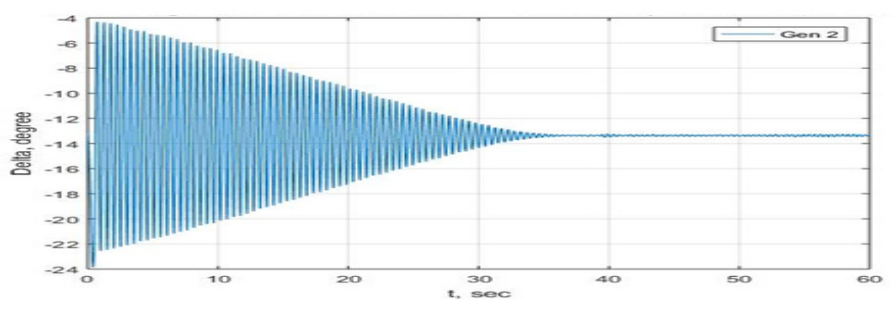

(b): Phase angle difference with line 3-4 removed

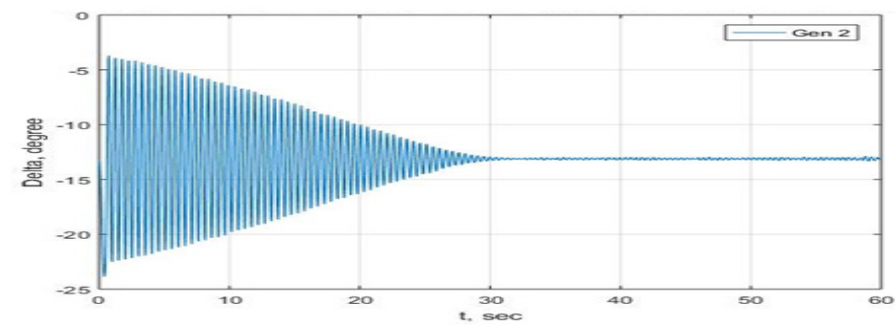

(c): Phase angle difference with line 4-5 removed

Figure 14: IEEE 5-bus transient stability assessment before the integration of UPFC

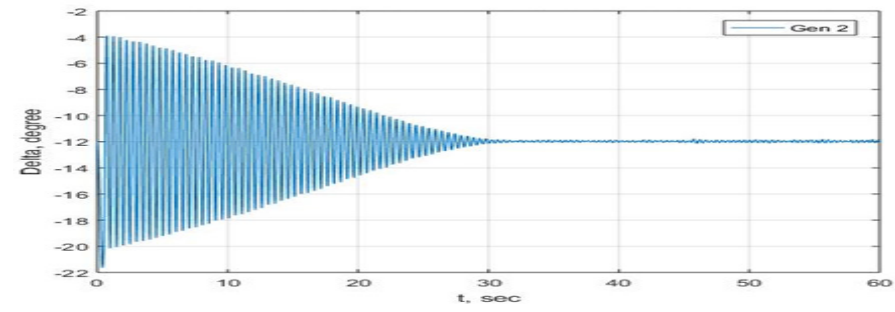

Figure 15: IEEE 5-bus transient stability assessment considering UPFC integration 


\section{Case 2: Transient Stability of The IEEE 30-Bus System}

In this subsection, the transient stability assessment of the standard IEEE 30-bus power network is carried out. The simulation results obtained when the critical load bus (bus 23) with the least eigenvalue is subjected to a fault are presented and discussed in this section. The N-1 criterion is also taken into consideration as explained for the IEEE 5-bus network. To determine the Critical Clearing Time (CCT) for the network, the transient stability assessment of the network is first performed using a small clearing time and increase in steps to determine the actual clearing time the network becomes unstable. This maximum clearing time at which the network becomes stable is taken as the CCT.

The dynamic responses obtained from the simulation before integrating the multi-UPFC are shown in Figures 16 (a) and (b). Based on the simulation results obtained, the Critical Clearing Time (CCT) of $0.01 \mathrm{~s}$ is obtained for the network. Figure 16 (a) shows the transient analysis when line $15-23$ is removed. The network regains transient stability after 30 seconds. Similarly, when line 23-24 is removed, the time taken to regain transient stability is 50 seconds, as presented in Figure 16 (b).
The most critical line based on the results presented in Table 2 is line 23-24. Based on the eigenvalue analysis results obtained, the line on which the series UPFC is placed is identified to be the one connecting buses 23 and 15 . Considering the N-1 contingency conditions, when a fault occurs at the most critical bus (bus 23), the most critical transmission line (the line connecting buses 23 and 24), as identified by the approach suggested in this study, is disconnected. For faults on bus 23 , the reactive power is increased in steps, and the critical line attached to it, based on $\mathrm{N}-1$ criterion is removed, and the transient stability analysed. The dynamic response obtained from the simulation is shown in Figure 17

It is evident from results obtained in Figure 17 that the inclusion of the multi-UPFC controller causes the network to regain transient stability faster than the time taken without the UPFC controller. Figure 17 confirms the improvement in time to 20 seconds from the base case of 50 seconds which it takes the system to regain transient stability when the critical line (line 2324 ) is disconnected. The result shows that by the integration of the FACTS device, the transient stability of the power system improves significantly. The improvement is evident in the timereduction of the distortion period, and this goes a long way at avoiding total system collapse during any major disturbance, usually faults.

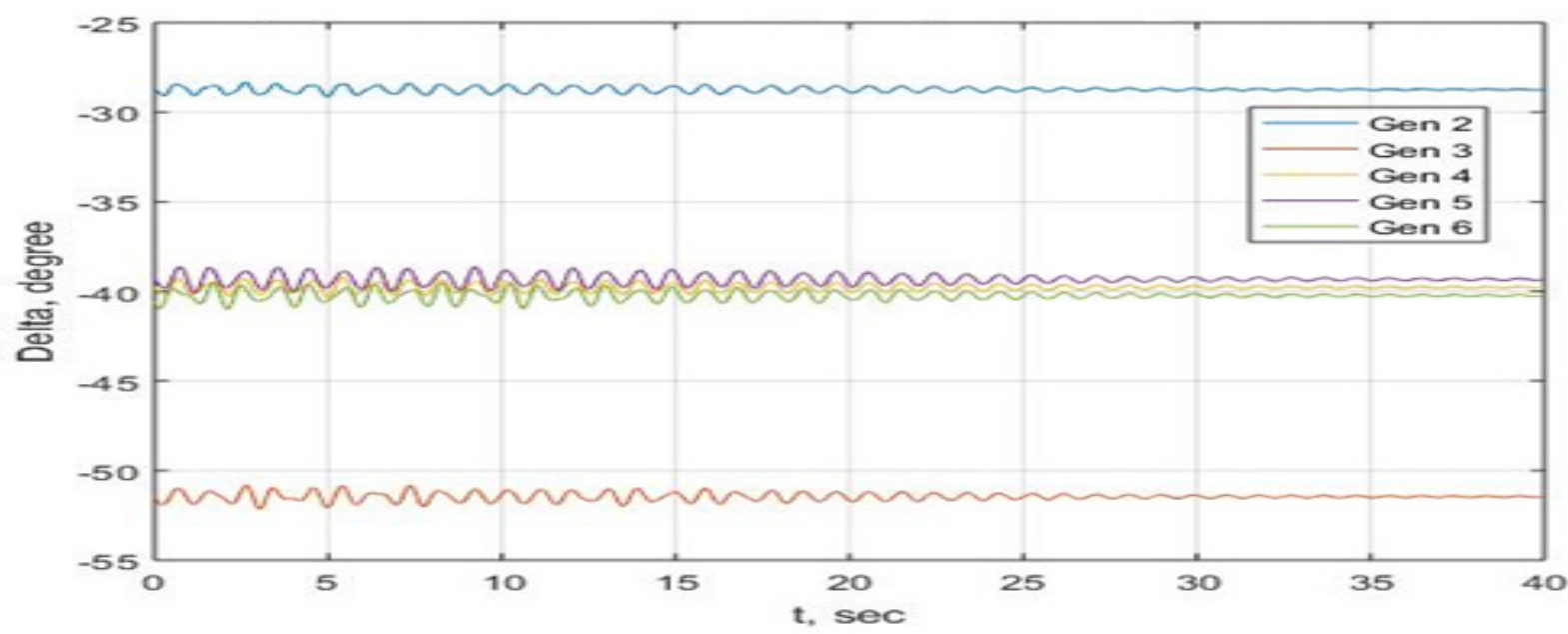

(a): Phase angle difference with line 15-23 removed

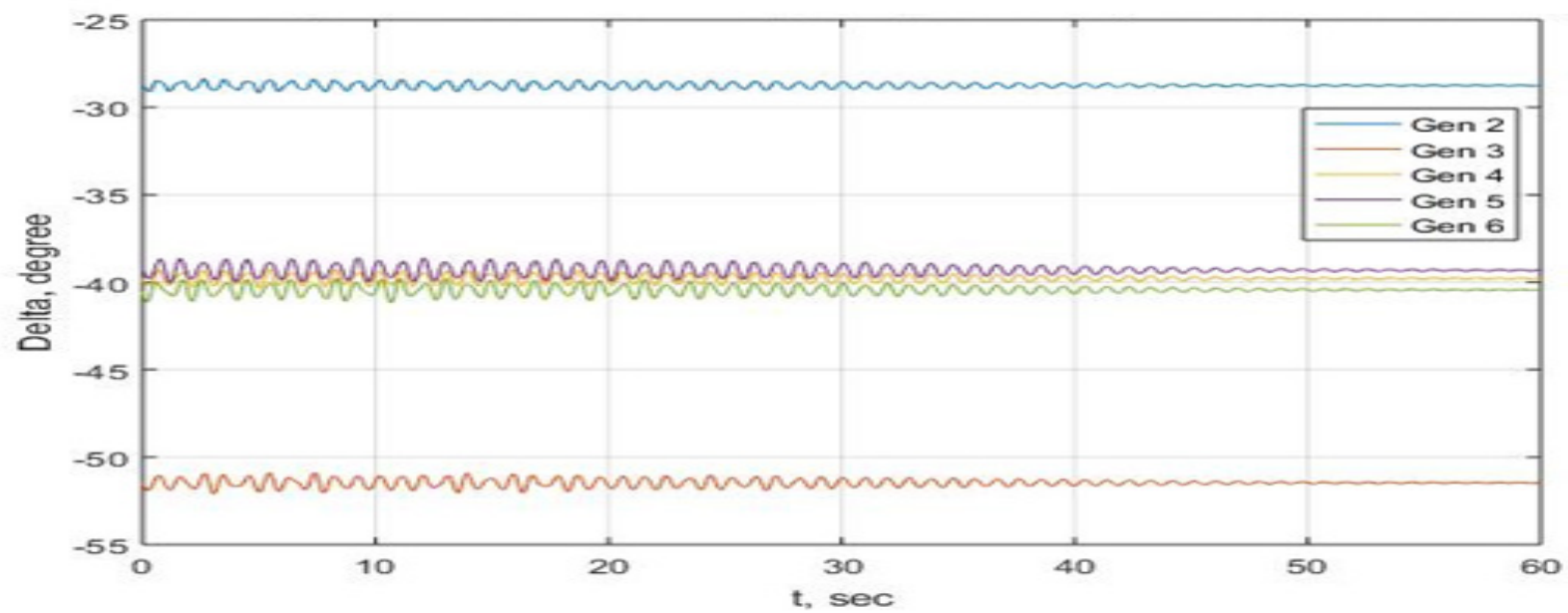

(b): Phase angle difference with line 23-24 removed

Figure 16: IEEE 30-Bus transient stability assessment before integrating UPFC 


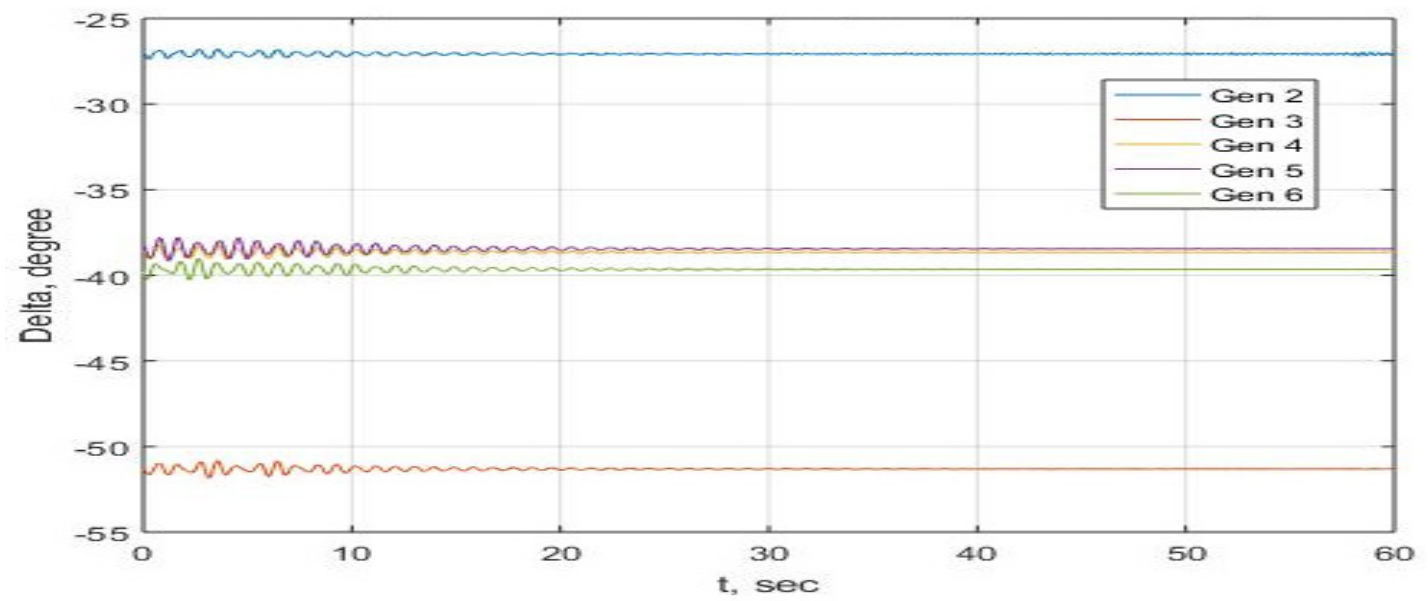

Figure 17: IEEE 30-bus transient stability assessment considering UPFC integration

\section{Case 3: The Nigerian 28-Bus System}

The same simulation procedures, as earlier discussed, are followed in this case. The total simulation time of 50 seconds and the clearing time of 0.08 second are considered for the simulation. The results obtained are presented in Figures 18 (a) and (b). By considering the $\mathrm{N}-1$ criterion, the transmission line connecting New Heaven to Onitsha (line 22-21) is first disconnected with reference to a fault occurring on the bus 22 and the simulation result obtained is shown in Figure 18 (a). The simulation result obtained when the transmission line connecting New Haven to Alaoji (22-23) is disconnected is shown in Figure 18 (b).

The base cases show that three generators are significantly out of synchronism with the others, with reference to the generator at Egin. The worst scenario is seen at a phase angle of $-10.75 \mathrm{o}$. The procedure is once again repeated after the integration of the UPFC to examine the impact of ingesting reactive power into the system through the UPFC.

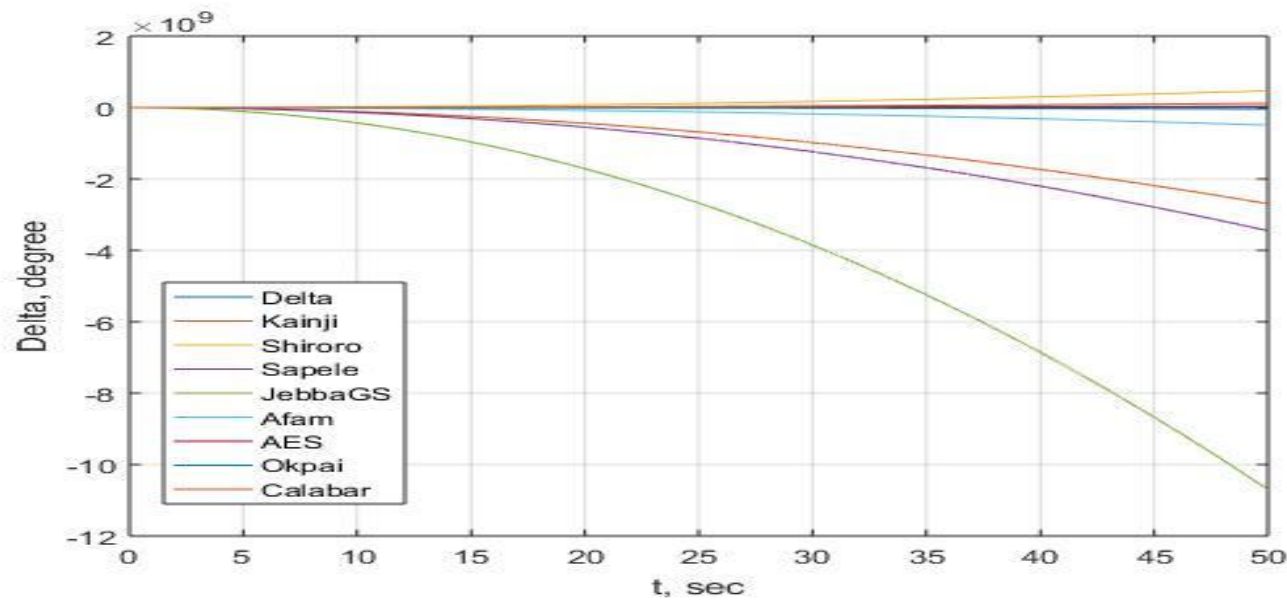

(a): Phase angle difference with line 21-22 removed

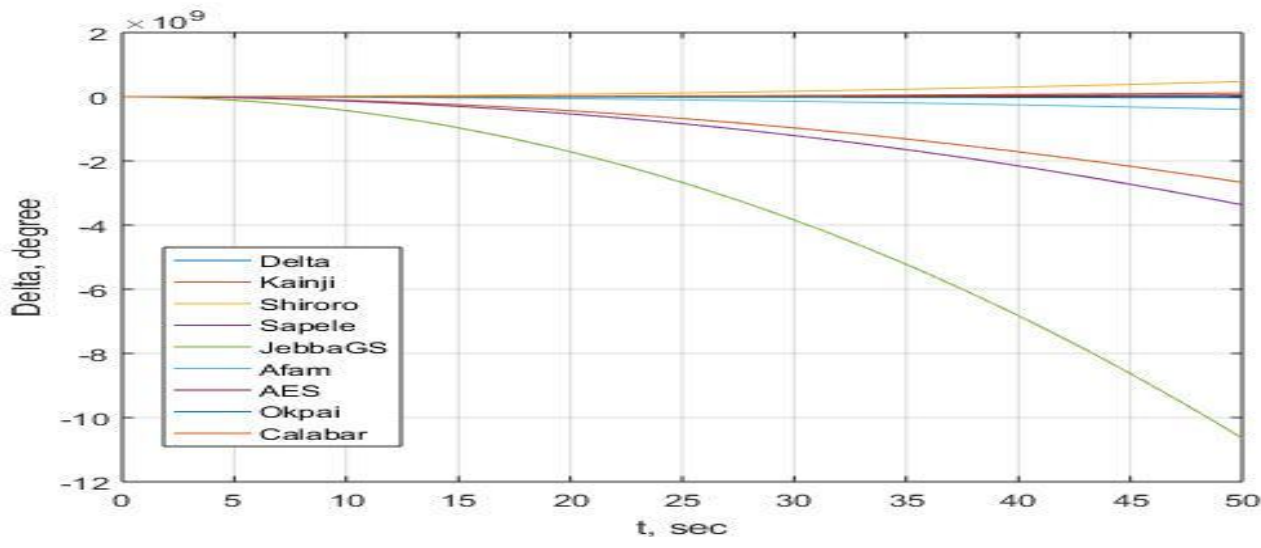

(b): Phase angle difference with line 22-23 removed

Figure 18: Nigerian 28-Bus Transient Stability Before Integrating FACTS Device 
From the results of the eigenvalue presented in Table 3, the placement of the UPFC is identified based on the magnitude of the eigenvalues of the network load buses. The bus with the least eigenvalue, within the network, is identified to be bus 22 (New Haven). The transmission line connecting this bus and the next ranked eigenvalue bus 23 (Alaoji) based on the approach suggested in this work is identified as the most critical transmission line in the system. In other words, the most critical line in the Nigerian 28-bus system is the transmission line connecting New Haven and Alaoji buses (bus 22- bus 23). This, therefore, suggests that from the worst scenario when a fault occurs, the line connecting buses 22 and 23 should be removed to clear the fault. Furthermore, as presented in Table 3, the next ranked eigenvalue bus, connected to the critical bus, after Alaoji bus (bus 23) is Onitsha bus (bus 21). This implies that the network can easily be reinforced by placing the UPFC controller in series with the line which connects the most critical bus 22 and bus 2 (New Haven and Onitsha).

The impact of the multi-UPFC when the transmission line connecting New Haven and Alaoji (bus 22- bus 23) is removed is shown in Fig. 19. The results show that two more generators are caused to get into synchronism to make it a total of eight generators with reference to the generator at Egbin. Only one generator was thrown out of synchronism, and this shows a notable improvement in the system by reinforcing with multiUPFC.

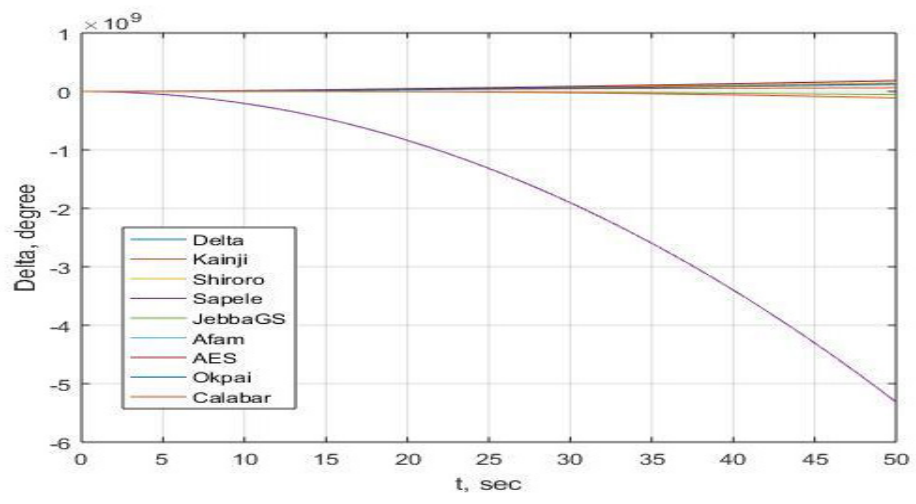

Figure 19: Transient stability for the phase angle difference with line 22-23 removed

When the generator bus at Sapele, which is out of synchronism with the critical line (line 22-23) is removed, a phase difference of -5.20 (as against -10.750 of the base cases) is obtained. This is evidence of the improved stability of the Nigerian 28-bus power network by reinforcing with the multi-UPFC controller.

\section{Conclusion}

In this paper, the influence of FACTS device integration on the assessment and enhancement of power system transient stability, in topologically weak power networks, have been presented. An extensive review of the existing studies on various approaches for solving the problem has been presented. The theoretical framework, as well as the mathematical formulations required for the analysis, are presented. Based on the mathematical formulations presented, a non-iterative based technique, which identifies the critical parts of the network where FACTS devices could be suitably located, for reinforcement of weak network, is suggested. The multi-UPFC is employed for this purpose in this study. The standard IEEE 5-bus and 30-bus, as well as the Nigerian 28-bus networks, are used as case studies in order to test the effectiveness of the method suggested in this study. The results obtained before and after the integration of UPFC are compared. From the comparison of results obtained, it is clearly revealed that the UPFC also has a great influence on the simulation times. More conclusively, this study has shown that the integration of FACTS devices into a power system is effective for improving transient stability when the system faults occur in any part of the system. It is shown, in this study, that the integration of FACTS Devices reduces, to the barest minimum, the total time taken for the system to regain balance and return to its normal operating conditions without experiencing system collapse.

\section{Conflict of Interest}

The authors declare no conflict of interest.

\section{Acknowledgment}

This research work and publication charge is fully funded by Covenant University Centre for Research, Innovation and Discovery (CUCRID).

\section{References}

[1] A. Halder, N. Pal, D. Mondal, "Transient Stability Analysis of a Multimachine Power System with TCSC Controller - A Zero Dynamic Design Approach," International Journal of Electrical Power and Energy Systems, 97, 51-71. 2018, doi:10.1016/j.ijepes.2017.10.030.

[2] C. Zambrano, S. Arango-Aramburo, Y. Olaya, "Dynamics of powertransmission capacity expansion under regulated remuneration," International Journal of Electrical Power and Energy Systems, 104, 924-932. 2019, doi:10.1016/j.ijepes.2018.07.029.

[3] A. AL Ahmad, R. Sirjani, Optimal placement and sizing of multi-type FACTS devices in power systems using metaheuristic optimisation techniques: An updated review, Ain Shams Engineering Journal, 2019, doi:10.1016/j.asej.2019.10.013.

[4] T.S. Shomefun, A. Ademola, C.O.A. Awosope, A.I. Adekitan, "Critical review of different methods for siting and sizing distributed-generators," Telkomnika (Telecommunication Computing Electronics and Control), 16(5), 2018, doi:10.12928/TELKOMNIKA.v16i5.9693.

[5] A.S. Alayande, A.A.G. Jimoh, A.A. Yusuff, "Reinforcement of Topologically Weak Power Networks Through Network Structural Characteristics Theory," International Journal of Emerging Electric Power Systems, 2018, doi:10.1515/ijeeps-2017-0219.

[6] T.H. Sikiru, A.A. Jimoh, Y. Hamam, J.T. Agee, R. Ceschi, "Classification of networks based on inherent structural characteristics," in Proceedings of the 2012 6th IEEE/PES Transmission and Distribution: Latin America Conference and Exposition, T and D-LA 2012, 2012, doi:10.1109/TDCLA.2012.6319055.

[7] K. Karthikeyan, P.K. Dhal, "Optimal Location of STATCOM based Dynamic Stability Analysis tuning of PSS using Particle Swarm Optimization," in Materials Today: Proceedings, 2018, doi:10.1016/j.matpr.2017.11.122.

[8] B. Vijay Kumar, V. Ramaiah, "Enhancement of dynamic stability by optimal location and capacity of UPFC: A hybrid approach," Energy, 2020, doi:10.1016/j.energy.2019.116464.

[9] K. Karthikeyan, P.K. Dhal, "A Hybrid BBO-DE optimization with Eigen value analysis based transient stability improvement by coordinated design of SVC," in Materials Today: Proceedings, 2018, doi:10.1016/j.matpr.2017.11.207.

[10] A.A. Jimoh, T.H. Sikiru, I.G. Adebayo, A.S. Alayande, "Influence of loading conditions in solving power system problems," in 2014 Australasian Universities Power Engineering Conference, AUPEC 2014 - Proceedings, 2014, doi:10.1109/AUPEC.2014.6966597.

[11] M. Tostado, S. Kamel, F. Jurado, An effective load-flow approach based on 
Gauss-Newton formulation, International Journal of Electrical Power and Energy Systems, 2019, doi:10.1016/j.ijepes.2019.06.006.

[12] M. Karimi, A. Shahriari, M.R. Aghamohammadi, H. Marzooghi, V. Terzija, Application of Newton-based load flow methods for determining steadystate condition of well and ill-conditioned power systems: A review, International Journal of Electrical Power and Energy Systems, 2019, doi:10.1016/j.ijepes.2019.05.055.

[13] H. Yang, F. Wen, L. Wang, "Newton-Raphson on power flow algorithm and Broyden method in the distribution system," in PECon 2008 - 2008 IEEE 2nd International Power and Energy Conference, 2008, doi:10.1109/PECON.2008.4762737.

[14] Modern Power Systems Analysis (Power Electronics and Power Systems) | Xi-Fan Wang, Yonghua Song, Malcolm Irving | download, Dec. 2020.

[15] T.H. Sikiru, A.A. Jimoh, J.T. Agee, "Inherent structural characteristic indices of power system networks," International Journal of Electrical Power and Energy Systems, 2013, doi:10.1016/j.ijepes.2012.11.011.

[16] T.E. Somefun, C.O.A. Awosope, A. Abdulkareem, A.S. Alayande, "Deployment of power network structural topology to optimally position distributed generator within distribution system," Journal of Engineering Science and Technology Review, 13(1), 2020, doi:10.25103/jestr.131.2.

[17] A.S. Alayande, A.A. Jimoh, A.A. Yusuff, "An alternative algorithm for solving generation-to-load matching and loss allocation problems," International Transactions on Electrical Energy Systems, 2017, doi:10.1002/etep.2347.

[18] A.S. Alayande, A.A. Jimoh, A.A. Yusuff, "Solution to network usage allocation problem in power networks," in 2016 IEEE International Conference on Renewable Energy Research and Applications, ICRERA 2016, Institute of Electrical and Electronics Engineers Inc.: 719-725, 2017, doi:10.1109/ICRERA.2016.7884428.

[19] A.S. Alayande, A.A. Jimoh, A.A. Yusuff, "Identification of critical buses and weak transmission lines using inherent structural characteristics theory," in Asia-Pacific Power and Energy Engineering Conference, APPEEC, 2016, doi:10.1109/APPEEC.2015.7380974.

[20] P.K. Gouda, A.K. Sahoo, P.K. Hota, "Optimal power flow including unified power flow controller in a deregulated environment," International Journal of Applied Engineering Research, 2015.

[21] A.O. Melodi, B.O. Akinloye, "Transmission Capacity Enhancement for Nigerian Power Transmission Grid using TCSC and UPFC," 8(2), 82-90, 2014.

[22] Akwukwuegbu IO, Nosiri OC, Agubor CK, Olubiwe M, "Comparative Power Flow Analysis of 28 and 52 Buses for 330KV Power Grid Networks in Nigeria Using Newton-Raphson Method," IJRERD International Journal of Recent Engineering Research and Development, Dec. 2020. 